\title{
DISTINCTION CERTIFICATION PROGRAM FOR ASSISTED INFUSIONAL THERAPY SERVICES - A COMPETITIVE EDGE
}

\author{
Adriana Freitas Silva1, ${ }^{1, \star}$ Lucianna Reis Novaes ${ }^{1}$, Geovana Magalhães Ferecini Tomasella ${ }^{1}$, Elizabeth Fernandes Reis ${ }^{1}$ \\ 1. Instituto Qualisa de Gestão - Health Services Accreditation, São Paulo (SP), Brazil. \\ *Corresponding author: seguranca@iqg.com.br
}

\section{BACKGROUND}

According to the World Health Organization (WHO), rheumatologic diseases are among the main causes of disability in the world. Therefore, the Instituto Qualisa de Gestão (IQG), in partnership with the Sociedade Brasileira de Reumatologia (SBR), developed the distinction certification of assisted infusional therapy services with the purpose of promoting, through the application of a systematic approach, the improving in the quality of care by reducing the variability in work processes. The objective of this work was to describe the perception of managers in relation to the cocreation process, scope of purpose and consequent certification of their services.

\section{METHODS}

The method consisted of three assessments in digital mode, within six months, with recorded approaches using the focus group technique. Perceptions were assessed by discourse analysis. Each assessment was conducted by a team of three healthcare professionals, being a moderator, an observer and a recorder. In the focus group with governance, the management model, measurement system, management for integrity and guidelines related to people, resources, access, processes, risks and communication were evaluated; in the operational management focus group, care coordination, pharmaceutical assistance, teamwork, organizational learning, biosafety, record structure and application of good practices were evaluated. In the last focus group, with the infusion team, the protocols, indicators and innovations were evaluated.

\section{RESULTS}

Discourse analysis allowed the identification of weaknesses related to structure, assistance, support to the patient and family, information system and evaluation of results. Discussions of standards guided the implementation of improvements in the care provided to patients, in the competitive advantage through the recognition of stakeholders and in the adequate recruitment and qualification of professionals directly or indirectly involved in care. From the certification, the implementation of innovative methods to consolidate safety in care practices was observed.

\section{CONCLUSION}

This process developed the service's managers and leaders to perceive challenges related to the implementation of the program requirements. The cocreation process allowed for the customization of the program, respecting the characteristics of the services, as well as the context in which they were inserted. This certification model helped to improve the quality of care by reducing variability, through the application of a systematic approach. Its main benefits were the strengthening of the clinical team cohesion to achieve favorable results, aligned with outcomes for specific rheumatologic diseases and generation of value for the patient.

\section{KEYWORDS}

Home infusion therapy, Rheumatic diseases, Certification. 